\title{
DOSSIER
}

\section{EL PODER DEL}

TESORO POR PARTE

DE LOS CONGRESOS

LOCALES: HACIA

NUEVAS PRÁCTICAS

José Said Sánchez*

RESUMEN: Este ensayo tiene como objetivo mostrar evidencia empírica sobre el ejercicio de la función presupuestaria por parte de los Congresos locales, específicamente en la fase de la aprobación. Si bien es cierto que en términos formales los gobernadores cuentan con más facultades para incidir en la política de gasto, el carácter reactivo de las legislaturas no las hace un jugador pequeño en el proceso. En términos generales, los Congresos están ejerciendo su poder del tesoro, incluso en contextos partidistas en los que menos se esperaría, a saber, los gobiernos unificados.

$$
\text { গ্ৰ }
$$

THE POWER OF THE TREASURY IN THE HANDS

\section{OF THE LOCAL CONGRESSES: NEW TRENDS}

ABSTRACT: This article will show empirical evidence regarding the exercise/practice of the budgetary role of the local Congresses, particularly during the approbatory phase. While/ Even though governors are given the skills to influence the budget, the legislature's explosive nature gives them an influential role in this process. The congresses are generally exercising their budgetary power, even in unexpected bipartisan contexts, namely, unified governments.

* Estudiante de doctrorado en Ciencia Social por El Colegio de México, Centro de Etudios Sociológicos; ganador del primer lugar en la segunda edición del Concurso de ensayo político Alonso Lujambio 2014, bajo el pseudónimo Sunproject. El jurado calificador estuvo integrado por Federico Estévez, Arturo Sánchez Gutiérrez, Jesús Silva-Herzog M., Rodolfo Vázquez y Horacio Vives. 
CITAM Derechos Reservados.

La reproducción total o parcial de este artículo se podrá hacer si el ITAM otorga la autorización previamente por escrito.

JOSÉ SAID SÁNCHEZ

ALABRAS CLAVE: función presupuestaria, gobierno dividido, gobierno unificado, congresos locales.

KEY WORDS: budgetary role, divided government, unified government, local congresses.

RECEPCIÓN: 2 de octubre de 2013. 


\section{EL PODER DEL TESORO POR PARTE DE LOS CONGRESOS LOCALES: HACIA NUEVAS PRÁCTICAS}

\section{Introducción}

\section{Un poder legislativo instituciona-} lizado es aquel que cuenta con autonomía, formalidad, uniformidad y complejidad. ${ }^{1}$ Lo anterior implica el funcionamiento de una rendición de cuentas horizontal efectiva, ${ }^{2}$ es decir, que el órgano legislativo difícilmente se somete a los deseos de su contraparte ejecutiva. Sin embargo, estas características a menudo sólo se pueden desarrollar bajo un contexto democrático. ${ }^{3}$ En retrospectiva, en el caso mexicano podemos identificar dos momentos que contrastan por el tipo de actividad que ha jugado el Poder Legislativo dentro de la política nacional. El primero se ubica durante el régimen autoritario, específicamente entre 1934 y 1997. En él, el Legislativo generalmente estuvo subordinado a los dictados del Ejecutivo. ${ }^{4}$ Las condiciones que permitieron semejante situación fueron el gobierno unificado, la disciplina partidista y el

${ }^{1}$ Samuel Patterson y Gary Copeland, "Parliaments in the Twenty-first Century", en Gary Copeland y Samuel Patterson (eds.), Parliament in the Modern World. Changing Institutions, 1994, Ann Arbor, University of Michigan Press, pp. 1-11.

${ }^{2}$ Guillermo O’Donnell, “Accountability horizontal: la institucionalización legal de la desconfianza política”, en Revista Española de Ciencia Política, núm. 11, 2004, pp. 11-33.

${ }^{3}$ Caroline Beer, "Assessing the Consequences of Electoral Democracy: Subnational Legislative Change in Mexico”, en Comparative Politics, vol. 33, núm. 4, julio, 2001, pp. 421-40.

${ }^{4}$ Aunque hay matices sobre esta idea. Véase Juan Molinar y Jeffrey Weldon, Los procedimientos legislativos en la Cámara de Diputados. 1917-1964, 2009, México, Miguel Ángel Porrúa. 
hecho de que el presidente fuera el líder indiscutible del partido. ${ }^{5} \mathrm{El}$ segundo momento, que llega hasta la actualidad, inició en 1997 con la inauguración de los gobiernos divididos en la Cámara de Diputados. ${ }^{6}$ Los principales efectos de este cambio cualitativo han sido tanto el rediseño institucional del Congreso ${ }^{7}$ como una mayor participación en la legislación. ${ }^{8}$

Pero, ¿qué sucede con los Congresos locales? En la dimensión partidista encontramos una diversidad en la integración de las legislaturas. Desde el primer gobierno dividido de Baja California en 1989 hasta la fecha, su número y sus variantes han aumentado. ${ }^{9}$ En la dimensión

${ }^{5}$ Jeffrey Weldon, “Las estrategias presidenciales con gobierno divido en México: 1917-1937”, en María Amparo Casar e Ignacio Marván (coords.), Gobernar sin mayoría. México 1867-1997, 2002, México, Taurus-CIDE, pp. 265-92; “The Legal and Partisan Framework of the Legislative Delegation of the Budget in Mexico", en Scott Morgenstern y Benito Nacif (eds.), Legislative Politics in Latin America, 2002, Cambridge, Cambridge University Press, pp. 377-410.

${ }^{6}$ Escenario en el que el partido del Ejecutivo no cuenta con el 50\% más uno de los escaños, véase Alonso Lujambio, "Estudio introductorio", en Alonso Lujambio (ed.), Poder Legislativo. Gobiernos divididos en la Federación Mexicana, 1996, México, UAM-IFE-Colegio Nacional de Ciencias Políticas y Administración Pública, pp. 9-31; “Gobiernos divididos en once estados de la federación mexicana, 1989-1997”, en Casar y Marván, op. cit., pp. 319-48.

${ }^{7}$ Luisa Béjar, "La (re)institucionalización del Poder Legislativo", en Revista Mexicana de Sociología, núm. 3, vol. 63, 2001, pp. 99-133.

${ }^{8}$ Luisa Béjar, “¿Quién legisla en México? Descentralización y proceso legislativo”, en Revista Mexicana de Sociología, 74, núm. 4, octubre-diciembre, 2012, pp. 619-47; María Amparo Casar, "Quince años de gobiernos sin mayoría en el Congreso mexicano", en Politica y gobierno, vol. XX, núm. 2, segundo semestre, 2013, pp. 219-63; "Los gobiernos sin mayoría en México: 1997-2006", en Política y gobierno, núm. 2, vol. XV, segundo semestre, 2008, pp. 221-70; Benito Nacif, "El fin de la presidencia dominante: la confección de las leyes en un gobierno dividido", en José Luis Méndez (coord.), Los grandes problemas de México. Políticas públicas, 2010, México, El Colegio de México, pp. 45- 83; "Las relaciones entre los poderes ejecutivo y legislativo tras el fin del presidencialismo en México", en Politica y gobierno, núm. 1, vol. XI, primer semestre, 2004, pp. 9-41.

${ }^{9}$ Los criterios para distinguir entre gobiernos unificados y divididos requieren ser analizados y esclarecidos, sobre todo con el aumento de las coaliciones electorales que han logrado ganar la gubernatura. Si en las coaliciones que lograron la victoria electoral sólo consideramos al partido con el mayor porcentaje de escaños, tenemos que en 2009, 16 estados estuvieron bajo gobiernos divididos y para 2014 esta cifra llegó a 21. Si consideramos el porcentaje de todos los partidos integrantes de la coalición, tenemos que en 2009 hubo 14 estados bajo gobierno dividido, cifra que se mantiene hasta 2014. Este aumento se detalla en Alonso Lujambio y Horacio Vives, El poder compartido. Un ensayo sobre la democratización mexicana, 2000, México, Océano; Alonso Lujambio, “Gobiernos divididos en once estados de la federación mexicana, 1989-1997", op. cit. 
formal, la evidencia ha mostrado la debilidad de los Congresos locales en términos institucionales. ${ }^{10} \mathrm{Al}$ respecto, una de las explicaciones sobre semejante situación destaca que los Congresos más institucionalizados se ubican en los estados con mayor competitividad electoral ${ }^{11}$ y pluralidad política legislativa. ${ }^{12}$ Además, en los medios y en la academia, se ha destacado que los gobernadores cuentan con mayor autonomía y poder tanto en relación con el Ejecutivo federal ${ }^{13}$ como con respecto a los poderes subnacionales (Legislativo y Judicial). ${ }^{14}$ En efecto, el aumento de las transferencias federales hacia las entidades federativas en las últimas dos décadas, los casos de gobiernos endeudados o la débil aplicación de la ley sobre el abuso de autoridad, por mencionar sólo algunos aspectos, recuerdan más la imagen de una democracia delega-

${ }^{10}$ Alonso Lujambio, "Los congresos locales: la precaria institucionalidad", en Robert Balkin (coord.), El poder Legislativo estatal en México, 2004, México, Universidad Estatal de Nueva York, pp. 169-87; Moisés Pérez, "La debilidad institucional de los congresos locales. Límites de la democratización mexicana y de las transformaciones de las relaciones EjecutivoLegislativo", en Andamios, vol. 5, núm. 10, abril, 2009, pp. 253-78; Khemvirg Puente, "Democratización, Congreso y Gasto Público en México. La lógica del pacto político presupuestal entre 1994-2010", Tesis de Doctorado en Ciencia Social, Centro de Estudios Sociológicos, El Colegio de México, 2011; Alejandra Ríos Cazáres, "Instituciones políticas estatales y presupuesto. Las causas de las discrecionalidad", en Estudios sobre Desarrollo Humano, 2009, México, PNUD, pp. 1-37.

${ }^{11}$ Caroline Beer, op. cit.

${ }^{12}$ Frederick Solt, "Electoral Competition, Legislative Pluralism, and Institutional Development: Evidence from Mexico's States”, en Latin American Research Review, vol. 39, núm. 1, 2004, pp. 155-67.

${ }^{13}$ Rogelio Hernández, El centro dividido. La nueva autonomía de los gobernadores, 2008, México, El Colegio de México; Leo Zuckermann, "Los señores feudales van a querer garantías", en Nexos, 1 de agosto de 2013 [consultado en junio de 2014. Disponible en http:/www.nexos. com.mx/?p=14391]; "Los nuevos virreyes", en Proceso, 28 de abril de 2004 [consultado en junio de 2014. Disponible en http://www.proceso.com.mx/?p=233027].

${ }^{14}$ Arturo Alvarado, "Los gobernadores y el federalismo mexicano", en Revista Mexicana de Sociología, vol. 58, núm. 3, julio-septiembre, 1996, pp. 39-71; Paola Gutiérrez, "El poder de los gobernadores en México 2001-2012: límites institucionales y políticos a nivel subnacional", Tesis de Doctorado en Investigación en Ciencias Sociales, FLACSO-México, 2013; Ríos Cázares, op. cit.; Fanny Sleman, "El poder presupuestal de los gobernadores en México (2000-2012): la influencia de las reglas presupuestales y del pluralismo legislativo", Tesis de Doctorado en Investigación en Ciencias Sociales, México, FLACSO-México, 2014. 
tiva ${ }^{15}$ que la de un régimen en el que funciona el sistema de pesos y contrapesos. ${ }^{16}$

Sin embargo, como veremos, el poder de los gobernadores no es absoluto en todas las áreas ni es igual en todos los estados. En una de las áreas más importantes, como lo es la política de gasto, existe un ejercicio activo de la función presupuestaria por parte de los Congresos locales, incluso en contextos donde menos se lo esperaría: el gobierno unificado. Lo anterior significa que el Congreso no apoya de manera mecánica la propuesta de gasto del gobernador ni es un órgano marginal, incluso cuando la evidencia muestra su debilidad en términos formales. En este ensayo no buscamos hacer una apología de los gobernadores ni minimizar su falta de rendición de cuentas, sino destacar la creciente actividad de los Congresos locales para determinar el rumbo del gasto en sus respectivas entidades. En términos más generales, estamos hablando de la activación incipiente del poder del tesoro a nivel local.

Para demostrar esta idea, ofrecemos evidencia empírica sobre la función presupuestaria de los Congresos locales. En primer lugar, señalamos algunas consideraciones sobre los regímenes subnacionales y las investigaciones en torno al gobierno dividido. En la segunda parte, mencionamos brevemente las particularidades de las finanzas estatales. Posteriormente, mostramos evidencia sobre el ejercicio de la función presupuestaria y hacemos un breve análisis comparativo de tres casos. Por último, exponemos algunas reflexiones que destacan el papel de los Congresos en la política de gasto, específicamente en la fase de la aprobación.

${ }^{15}$ Guillermo O'Donnell, "Revisando la democracia delegativa", en Casa del Tiempo, núm. 31, mayo, 2010, pp. 2-8; “La otra institucionalización”, en Política y gobierno, núm. 3, vol. 2, 1996, pp. 219-44.

${ }^{16}$ En este sentido, para el caso argentino a nivel federal, también se ha tratado de matizar el concepto de democracia delegativa y mostrar que el Congreso ha tenido un papel dentro del proceso presupuestario. Véase Mark Jones, "Political Institutions and Public Policy in Argentina. An Overview of the Formation and Execution of the National Budget", en Stephan Haggard y Mathew McCubbins (eds.), Presidents, Parliaments, and Policy, 2001, Cambridge, Cambridge University Press, pp. 149-82. 


\section{Régimen subnacional y gobiernos divididos}

Analizar la conducta de los Congresos locales implica tener una idea del régimen político en el cual se desenvuelven. En este sentido, las investigaciones sobre los regímenes subnacionales muestran que, en las democracias de la tercera ola, la transición ha tenido un impacto diferencial sobre las unidades subnacionales, ${ }^{17}$ las cuales cumplen en diferente grado con los requisitos procedimentales de una democracia. Aunque los estudios se han enfocado en explicar la persistencia de los regímenes subnacionales no democráticos dentro de una democracia nacional, consideramos que también es importante conocer las características de las funciones que ejerce el poder Legislativo en estos contextos. De manera general, se supondría que en aquellos regímenes subnacionales menos democráticos, el poder Legislativo goza de escaso grado de institucionalización y autonomía frente al Ejecutivo, frente a aquellos regímenes subnacionales más democráticos (idea que ha sido demostrada para algunos casos). ${ }^{18}$

En este sentido y de manera más específica, el tipo de gobierno (unificado o dividido) ha sido utilizado como variable explicativa de la conducta del poder Legislativo. Al respecto, el modelo de party government, desarrollado durante la primera mitad del siglo XX, consideró al gobierno unificado como la mejor opción para fomentar un gobierno eficaz. En síntesis, este modelo considera al partido político como un instrumento que establece un puente entre la rama Legislativa y Ejecutiva, lo que

\footnotetext{
${ }^{17}$ Wayne Cornelius, "Subnational Politics and Democratization: Tensions between Center and Periphery in the Mexican Political System", en Wayne Cornelius, Todd Eisenstadt y Jane Hindley (eds.), Subnational Politics and Democratization in Mexico, 1999, Boulder (CO), Lynne Rienner, US-Mexico Contemporary Perspectives Series, 13, pp. 3-16; Carlos Gervasoni, "Democracia, autoritarismo e hibridez en las provincias argentinas: la medición y causas de los regímenes subnacionales", en Journal of Democracy en Español, vol. 3, 2011, pp. 75-93; Edward Gibson, "Control de límites: autoritarismo subnacional en países democráticos", en Desarrollo Económico, vol. 47, núm. 186, julio-septiembre, 2007, pp. 163-91; Agustina Giraudy, "The Politics of Subnational Undemocratic Regime Reproduction in Argentina and Mexico", en Journal of Politics in Latin America, 2, 2010, pp. 53-84; Richard Snyder, "After the State Withdraws: Neoliberalism and Subnational Authoritarian Regimes in Mexico", en Cornelius, Eisenstadt \& Hindley, op. cit., pp. 295-341.

${ }^{18}$ Caroline Beer, op. cit.; Frederick Solt, op. cit.
} 
permite generar una acción coordinada y homogénea entre ambas. Además, el presidente ejerce una función de liderazgo que le facilita coordinar la acción de los legisladores que pertenecen a su partido. ${ }^{19}$ Aunque existe un sistema de división de poderes y de pesos y contrapesos, el partido político armoniza a las ramas separadas del gobierno. En efecto, lo anterior implica que el partido en el gobierno tenga la mayoría de escaños y que el presidente sea reconocido como el líder. Bajo tales condiciones, el presidente es quien determina las acciones del Congreso y logra obtener el apoyo para sus políticas: él es el policy maker. ${ }^{20}$

Sin embargo, cuando ambas ramas se encuentran en manos de partidos diferentes, los efectos establecidos por el modelo del party government no son tan claros. Bajo un gobierno dividido, el partido político del presidente no puede, o tiene más obstáculos para, coordinar la acción entre ambas ramas. Por lo tanto, a la división de poderes y al sistema de pesos y contrapesos que existen en un régimen democrático, hay que añadir la división política. ${ }^{21}$ Debido a la legitimidad democrática dual, derivada del hecho de que ambos poderes son elegidos mediante el voto popular, ${ }^{22}$ es más probable que bajo gobiernos divididos ambas ramas tiendan a proclamarse como los verdaderos representantes de la voluntad popular, lo que podría llevar a escenarios de parálisis política. ${ }^{23}$

Otras variables explicativas son sin duda el sistema de partidos y la disciplina partidista. ${ }^{24}$ En términos generales, un sistema multipar-

${ }^{19}$ James Sundquist, "Needed: a Political Theory of the New Era of Coalition Government in the United States", en Political Science Quarterly, 103, 1988, pp. 613-35.

${ }^{20}$ Ibid.

${ }^{21}$ En términos de Cox y McCubbins, por un lado tendríamos la división formal de poderes y, por el otro, la división de propósitos (actores políticos con diversos intereses que controlan puntos de veto distintos). Véase Gary Cox y Mathew McCubbins, "The Institutional Determinants of Economic Policy Outcomes”, en Haggard \& McCubbins, op. cit., pp. 21-63.

${ }^{22}$ Juan Linz, "Democracia presidencial o parlamentaria. ¿Qué diferencias implican?”, en Juan Linz y Arturo Valenzuela (comps.), La crisis del presidencialismo. El caso de América Latina, 1998, Madrid, Alianza, pp. 25-143.

${ }^{23}$ Aunque esto no es condición suficiente. Al respecto, véase Charles Jones, Separate but Equal Branches. Congress and the Presidency, 1995, New Jersey, Chatham House, p. 12.

${ }^{24}$ Scott Mainwaring y Mathew Shugart, "Presidencialismo y sistema de partidos en América Latina", en Scott Mainwaring y Mathew Shugart, Presidencialismo y democracia en América Latina, 2002, Bs. As., Paidós, pp. 255-94. 
tidista fragmentado genera más dificultades en la relación EjecutivoLegislativo, ya que el primero difícilmente logra una mayoría en el Congreso. Aunque el presidente puede contar con amplios poderes formales, puede estar limitado para modificar el status quo si no cuenta con una mayoría o si tiene serias dificultades para lograr una coalición. En el caso opuesto, puede contar con limitados poderes formales, pero si su partido tiene la mayoría en el Congreso y los legisladores son disciplinados, sus políticas podrían ser respaldadas. Este escenario corresponde a los gobiernos prí́stas durante buena parte del siglo XX.

A lo anterior hay que añadir las características específicas del juego bilateral de veto que existen en los sistemas presidenciales de América Latina. Una de ellas es la asimetría, ya que el Ejecutivo es quien hace el primer movimiento. ${ }^{25}$ Lo anterior implica que el presidente tiene un papel proactivo, en tanto que la legislatura desempeña uno reactivo. El primero, en mayor medida, tiene la capacidad para iniciar la legislación y colocar la agenda; por su parte, la legislatura reacciona modificando y enmendado tales propuestas. Es importante destacar que el hecho de que la Legislatura tenga un papel reactivo en esta relación no implica que su actividad carezca de importancia dentro de la policy-making. ${ }^{26}$

Por lo tanto, a partir de lo anterior se esperaría que bajo gobiernos divididos el Congreso ejerciera de manera activa la función presupuestaria frente a los gobiernos unificados. Como acabamos de señalar, esta idea se apoya en el supuesto de que existen altos grados de disciplina partidista entre los legisladores; en el caso mexicano, la disciplina se explica, entre otras cosas, por la prohibición de la reelección consecutiva y, en su momento, por la capacidad del presidente para determinar el futuro político de los legisladores. ${ }^{27}$ En el caso de los regímenes

${ }^{25}$ Gary Cox y Scott Morgenstern, "Epilogue: Latin America's Reactive Assemblies and Proactive President", en Morgenstern \& Nacif, op. cit., pp. 446-68.

${ }^{26}$ Esto ha sido propuesto y demostrado para algunos países de América Latina, véase Morgenstern y Nacif, op. cit., así como Gabriel Negretto, “¿Gobierna solo el presidente? Poderes de decreto y diseño institucional en Brasil y Argentina”, en Desarrollo Económico, vol. 42, núm. 167, octubre-diciembre, 2002, pp. 377-404.

${ }^{27}$ Benito Nacif, "Para comprender la disciplina de partido en la Cámara de Diputados de México: el modelo del partido centralizado", en Foro Internacional, núm. 167, 2002, pp. 5-38; "La no reelección consecutiva y la persistencia del partido hegemónico en la Cámara de Diputa- 
subnacionales, los gobernadores poseen amplios recursos para determinar el futuro político de los legisladores locales de su partido, ${ }^{28}$ por lo que también se esperarían altos grados de disciplina. Entonces, bajo gobiernos unificados los legisladores del partido del gobernador tendrán pocos incentivos para modificar el proyecto de egresos; en contraparte, bajo gobiernos divididos, dada la disciplina de la oposición, el gobernador tendrá más dificultades para encontrar apoyo, lo que se reflejará en mayores modificaciones al proyecto de presupuesto de egresos.

Pero, ¿empíricamente existen diferencias entre los gobiernos unificados y divididos? A pesar de los diversos estudios, aún no se llega a resultados contundentes. Por ejemplo, en el caso de la legislación en Estados Unidos, los hallazgos son en cierta medida contradictorios. Por un lado, se ha mostrado que no hay diferencia significativa entre los gobiernos divididos y los unificados en relación con la producción de legislación importante. ${ }^{29}$ Por el otro lado, existe evidencia que muestra lo contrario o se han matizado los hallazgos. ${ }^{30}$

En el caso mexicano existe un poco más de contraste. En lo que respecta a la legislación, se ha demostrado que con la llegada de los gobiernos divididos la actividad por parte de la Cámara de Diputados

dos de México", en Francisco Gil y Rogelio Hernández (coords.), Los legisladores ante las reformas políticas de México, 2001, México, El Colegio de México, pp. 83-128.

${ }^{28}$ José Cheibub, Argelina Figuereido y Fernando Limongi, "The Politics of Federalism in Brazil: The Role of Governors in the Brazilian Congress", presentado en el Seminar on Taxation Perspectives: A Democratic Approach to Public Finance in Developing Countries, 2002, Institute for Development Studies, University of Sussex; Joy Langston, "Governors and 'Their' Deputies: New Legislative Principals in Mexico”, en Legislative Studies Quarterly, vol. XXXV, núm. 2, mayo, 2010, pp. 235-58; Scott Mainwaring y David Samuels, "Federalism, Constraints On The Central Government, and Economic Reform in Democratic Brazil", en Working Paper no. 271, 1999, The Helen Kellogg Institute for International Studies.

${ }^{29}$ David Mayhew, Divided We Govern. Party Control, Lawmaking and Investigations, 1946-1990, 1991, New Haven, Yale University Press.

${ }^{30}$ George Edwards, Andrew Barret y Jeffrey Peake, "The Legislative Impact of Divided Government", en American Journal of Political Science, vol. 41, núm. 2, abril, 1997, pp. 545-63; William Howell, et. al., "Divided Government and the Productivity of Congress, 1945-1994", en Legislative Studies Quarterly, vol. XXV, núm.2, mayo, 2000, pp. 285-312; Sean Kelly, "Divided We Govern? A Reassessment", en Polity, vol. 25, núm. 3, verano, 1993, pp. 475-84. La revisión de estos estudios muestra que utilizan diferentes formas para operacionalizar la variable dependiente (legislación importante), lo que podría explicar sus distintos resultados. 
ha aumentado de manera notable y que no existe (hasta el momento) evidencia de parálisis política, ${ }^{31}$ aunque hay quienes señalan que en tales contextos las reformas estructurales se han visto limitadas. ${ }^{32}$ También se ha destacado el efecto sobre la normatividad interna, principalmente al dotar de un cariz plural a la integración de los órganos de gobierno. ${ }^{33}$ En el tema de la función presupuestaria, se ha observado que la Cámara de Diputados ha realizado modificaciones al proyecto del Presupuesto de Egreso de la Federación de manera constante. ${ }^{34}$

\section{El presupuesto de egresos}

En términos generales, el proceso presupuestario comprende cuatro etapas: elaboración, aprobación, ejecución y control del gasto. ${ }^{35}$ La participación de la Legislatura abarca la mayoría de estas etapas: tiene un papel tanto en la planeación presupuestal como en las asignaciones de gasto (ex ante); y también en las fases de la información financiera, la auditoria y la evaluación externa del proceso del presupuesto (expost) ${ }^{36}$

${ }^{31}$ Luisa Béjar, “¿Quién legisla en México? Descentralización y proceso legislativo”, op. cit.; María Amparo Casar, "Quince años de gobiernos sin mayoría en el Congreso mexicano", op. cit.; Benito Nacif, "El fin de la presidencia dominante: la confección de las leyes en un gobierno dividido", op. cit.

${ }^{32}$ VV. AA., "Gobernabilidad democrática en México: más allá de la captura del Estado y la polarización social”, 2007, México, Banco Mundial; Rogelio Hernández, "La relación entre poderes. Las reformas posibles", en Arturo Alvarado (ed.), México: democracia y sociedad, 2013, México, Tribunal Electoral del Poder Judicial de la Federación-El Colegio de México, pp. 169-90.

${ }^{33}$ Luisa Béjar, "La (re)institucionalización del Poder Legislativo", op. cit.

${ }^{34}$ Enrique Carpio, “Aprobación de las finanzas públicas y cambio político en dos gobiernos sin mayoría en México (1997-2006)", en Ricardo Espinoza y Jeffrey Weldon (coords.), El Congreso de la democracia, 2010, México, Senado de la República-AMEP, pp. 227-51; Laura Sour, "Pluralidad y transparencia en el proceso de aprobación presupuestal al interior de la Cámara de Diputados", en Cuadernos de Trabajo, 2006, CIDE.

${ }^{35}$ Benito Nacif, Para entender el Poder Legislativo de los Estados Unidos Mexicanos, 2005, México, Nostra.

${ }^{36}$ Rick Stapenhurst, "La legislatura y el presupuesto", en Rick Stapenhurst, Riccardo Pelizzo, David Olson y Lisa von Trapp (eds.), Fiscalización legislativa y presupuesto. Una perspectiva mundial, 2009, Colombia, Instituto del Banco Mundial, pp. 49-63. 
No obstante, esta participación varía entre países. ${ }^{37}$ Por ejemplo, en los sistemas presidenciales la legislatura tiene un papel más significativo frente a los sistemas parlamentarios y semipresidenciales. ${ }^{38}$ Además, se han detectado formas diversas de vigilancia (oversight) en la etapa ex post. ${ }^{39}$

Como el lector habrá notado, en este ensayo nos concentramos en la parte de la aprobación. Pero antes de continuar, es preciso dejar en claro ciertas cuestiones analíticas sobre la función presupuestaria del poder Legislativo. Por un lado, hay quienes consideran que la facultad para imponer contribuciones y aprobar el gasto público se ubica dentro de función legislativa, distinguiéndola de la función de control (oversight). ${ }^{40}$ Por el otro lado, están quienes integran la función presupuestaria dentro de la de control. ${ }^{41}$ En nuestro caso, sólo distinguiremos entre control ex ante y control ex post, ubicando a la función presupuestaria en la primera etapa.

En México, el Presupuesto de Egresos de la Federación (PEF) es el documento jurídico y financiero en el que se determina el monto y destino de los recursos económicos del gobierno federal durante un año (año fiscal). La elaboración del proyecto está a cargo del Ejecutivo, específicamente de la Secretaría de Hacienda y Crédito Público (SCHP). ${ }^{42}$

${ }^{37}$ Para un estudio sobre las características del proceso presupuestario en México en términos comparativos véase Gerónimo Gutiérrez, Alonso Lujambio y Diego Valadés, El proceso presupuestario y las relaciones entre los órganos de poder. El caso mexicano en perspectiva histórica y comparada, 2001, México, UNAM.

${ }^{38}$ Joachim Wehner, "Evaluar el poder del dinero: índice de instituciones legislativas de presupuesto", en Stapenhurst, Pelizzo, Olson y von Trapp, op. cit., pp. 79-101.

${ }^{39}$ Mathew McCubbins y Thomas Schwartz, "Congressional Oversight Overlooked: Police Patrols versus Fire Alarms", en American Journal of Political Science, 28, 1984, pp. 167-79.

${ }^{40}$ María Amparo Casar, María Marván y Khemvirg Puente, "La rendición de cuentas y el Poder Legislativo", en Mauricio Merino, Sergio López y Guillermo Cejudo (coords.), La estructura de la rendición de cuentas en México, 2010, México, Instituto de Investigaciones Jurídicas-CIDE, pp. 331-405.

${ }^{41}$ Arturo Alvarado, "El Congreso mexicano", en Ilán Bizberg y Lorenzo Meyer (coords.), Una historia contemporánea de México: las instituciones, 2009, México, Océano, pp. 119-61.

${ }^{42}$ El calendario presupuestario inicia con la entrega al Congreso de las primeras proyecciones macroeconómicas realizadas por la SHCP, a más tardar el 1 de abril. Entre junio y julio las secretarías comienzan a redactar sus propuestas de presupuesto, las cuales entregan a la SHCP. En julio, ésta comunica los topes presupuestales por sector a las secretarías; en agosto, la SHCP integra la propuesta del presupuesto y se realizan revisiones finales. La formulación 
Por su parte, la Cámara de Diputados tiene la facultad exclusiva para su aprobación. En este sentido hablamos de la función presupuestaria: la facultad para aprobar el presupuesto de egresos, previo examen, análisis $\mathrm{y}$, en su caso, modificación ${ }^{43}$ del proyecto enviado por el Ejecutivo; sin embargo, la facultad para hacer modificaciones tiene límites. Por ejemplo, la Cámara de Diputados no puede cambiar la estructura programática que el Ejecutivo somete al Congreso en el mes de junio, y no puede negar el financiamiento de gastos que el Ejecutivo está obligado a hacer legal o constitucionalmente, como las transferencias federales y el gasto en fondos y programas sociales. ${ }^{44}$ No obstante, el hecho de que la Cámara de Diputados cuente con esta facultad no nos dice nada sobre las características de su ejercicio, específicamente, para hacer modificaciones al proyecto del PEF.

Por obvias razones (gobierno unificado, disciplina partidista y el presidente como líder del partido), durante el régimen autoritario, el Ejecutivo introdujo el presupuesto de egresos a tiempo y la Cámara de Diputados aprobó el proyecto sin enmiendas. ${ }^{45}$ En otras palabras, la función presupuestaria estuvo ausente en la práctica, sobre todo entre 1978 y 1994; empero, hay evidencia que muestra que a mediados de los 80 este órgano comenzó a realizar algunas modificaciones en los montos del proyecto del $\mathrm{PEF},{ }^{46}$ actividad que a partir de 1997 se

del proyecto termina con la entrega del Paquete Económico al Congreso, el 8 de septiembre. Este paquete está integrado por: un análisis del marco macroeconómico, una propuesta de Ley de Ingresos y una propuesta de Decreto de Presupuesto de Egresos. La fecha límite de aprobación es el 15 de noviembre, pero en la práctica se extiende hasta el 31 de diciembre. Cuando inicia una nueva administración, el Ejecutivo puede enviar el proyecto hasta el 15 de diciembre y el Congreso puede aprobarlo hasta el 31 de diciembre. Véase Benito Nacif, Para entender el Poder Legislativo, op. cit.

${ }^{43}$ En 2004 se llevó a cabo una Reforma constitucional que aclaró la facultad de la Cámara de Diputados para modificar el proyecto, aunque en la práctica ya se realizaba esta actividad.

${ }^{44}$ Estudio de la OCDE sobre el proceso presupuestario en México, 2009, en OECD Journal of Budgeting, suplemento 1.

${ }^{45}$ Jeffrey Weldon, "The Legal and Partisan Framework of the Legislative Delegation of the Budget in Mexico", op. cit.

${ }^{46}$ Alberto Díaz-Cayeros y Beatriz Magaloni, “Autoridad presupuestal del poder legislativo en México: una primera aproximación”, Política y gobierno, vol. V, núm. 2, segundo semestre, 1998, pp. 503-28. 
vuelve constante y notable. ${ }^{47}$ Ante ello y dada la diversidad partidista de las legislaturas estatales, surge la curiosidad por saber qué es lo que sucede en este nivel.

Al respecto, a nivel subnacional se han medido las capacidades institucionales, tanto de los Congresos como de los gobernadores, para incidir en la política presupuestaria. Los resultados muestran importantes asimetrías. Los Congresos destacan por sus limitadas capacidades organizacionales e institucionales para influir en el presupuesto de egresos. ${ }^{48}$ En el tema de la oversight burocrática (control ex post), se ha detectado que su amplitud y profundidad depende de los intereses y de los recursos con los que cuentan los legisladores para llevarla a cabo. ${ }^{49}$ Respecto al poder de los gobernadores, se considera que sus facultades legislativas son fuertes y homogéneas, lo que les permite imponer su agenda presupuestal. ${ }^{50}$ En síntesis, a nivel subnacional, los estudios destacan la debilidad institucional de las legislaturas y el predominio de los gobernadores. No obstante, como ya hemos señalado, una parte de esta asimetría es inherente al juego bilateral de veto de los sistemas presidenciales de América Latina, en el que el Ejecutivo es quien hace el primer movimiento. A nivel subnacional y en el tema presupuestario la situación no es distinta: el Ejecutivo es proactivo y el Legislativo es reactivo. El carácter reactivo del Legislativo no implica que sea un actor sin importancia. Además, el tipo de relación no sólo depende del aspecto institucional, sino también del apoyo que el Ejecutivo logra en el Congreso. ${ }^{51}$ Por lo tanto, es preciso complementar este diagnóstico insti-

${ }^{47}$ Enrique Carpio, op. cit.; Laura Sour, op. cit.

${ }^{48}$ Khemvirg Puente, op. cit; Ríos Cázares, op. cit.

${ }^{49}$ Alejandra Ríos Cazáres, "The Circuitous Path of Democracy: Legislative Control of the Bureaucracy in presidential regimes. The Case of Mexico", Tesis de Doctorado en Ciencia Política, University of California, San Diego, 2010.

${ }^{50}$ Fanny Sleman, "El poder presupuestal de los gobernadores en México", en Revista de Investigación Scientia Educere, núm. 2, año 1, 2012, pp. 1-9; "El poder presupuestal de los gobernadores en México (2000-2012): la influencia de las reglas presupuestales y del pluralismo legislativo", op. cit.; Fanny Sleman y Álvaro López, "Los efectos de los diferentes tipos de reconducción presupuestal en las entidades federativas de México", en Gestión y estrategia, núm. 3, enero/junio, 2013, pp. 81-93; Ríos Cazáres, "Instituciones políticas estatales y presupuesto, op. cit.

${ }^{51}$ Gary Cox y Scott Morgenstern, op. cit.; Scott Morgenstern, "Explaining Legislative Politics in Latin America", en Morgenstern y Nacif, Legislative Politics in Latin America, op. cit., pp. 413-45. 
tucional con evidencia empírica, ya que se sabe poco sobre su ejercicio y sus características. ${ }^{52}$

Medir el ejercicio de la función presupuestaria requiere considerar ciertas características de las finanzas estatales. La principal es que alrededor del $90 \%$ de los ingresos de los estados proviene de las transferencias federales, aunque el porcentaje entre estados varía. ${ }^{53} \mathrm{Como}$ el nombre lo indica, se trata de recursos transferidos de un orden de gobierno a otro para financiar las necesidades de gasto. Estos recursos se integran, principalmente, por las participaciones y por las aportaciones. ${ }^{54}$ Grosso modo, las participaciones son recursos soberanos de las entidades y municipios, por lo que la federación no establece cómo se deben gastar. Incluso se pueden utilizar para el pago de obligaciones financieras contraídas por estados y municipios (previa autorización de los Congresos estatales). Por otro lado, las aportaciones no se pueden modificar, ya que son recursos etiquetados para áreas establecidas. Dadas las fórmulas que existen para determinar el monto de las participaciones y de las aportaciones, éstas representan porcentajes distintos en los ingresos de cada estado. En la gráfica 1 podemos ver las diferencias entre estados respecto a las aportaciones que reciben.

${ }^{52}$ Aunque Sleman y Rodríguez muestran evidencia, ambas se basan en la Encuesta de Expertos en Política Estatal en México, 2001-2012, elaborada por Flacso-México. Lo anterior implicó una aproximación indirecta al objeto de estudio; sin demeritar su aporte, en nuestro caso analizamos de manera directa los dictámenes del proyecto de presupuesto de egresos para cada estado. Además, Sleman se concentró en medir el poder presupuestario del gobernador; Gutiérrez analizó la capacidad del poder Legislativo y Judicial para controlar al gobernador; y nosotros nos enfocamos en la función presupuestaria ejercida por parte del Congreso.

${ }^{53}$ Horacio Sobarzo, "Relaciones fiscales intergubernamentales en México: evolución reciente y perspectivas", en Problemas del desarrollo, vol. 40, núm. 156, enero-marzo, 2009, pp. 11-28; Jorge Chávez y Fausto Hernández (coords.), El México del 2013. Hacia una reforma del federalismo fiscal, 2012, México, Centro de Estudios Espinosa Yglesias.

${ }^{54}$ A partir de 1980, con la creación del Sistema Nacional de Coordinación Fiscal, el gobierno federal se encarga de recaudar impuestos como el Impuesto al Valor Agregado (IVA) y el Impuesto sobre la Renta (ISR). Los estados delegaron esta capacidad recaudatoria al gobierno federal a cambio de recibir participaciones federales (ramo 28). Ahora sólo recaudan impuestos sobre nómina y participan en la recaudación del impuesto especial sobre producción y servicios. 
GRÁFICA 1

\section{Porcentaje promedio de aportaciones con respecto a ingresos totales, 2000-2012}

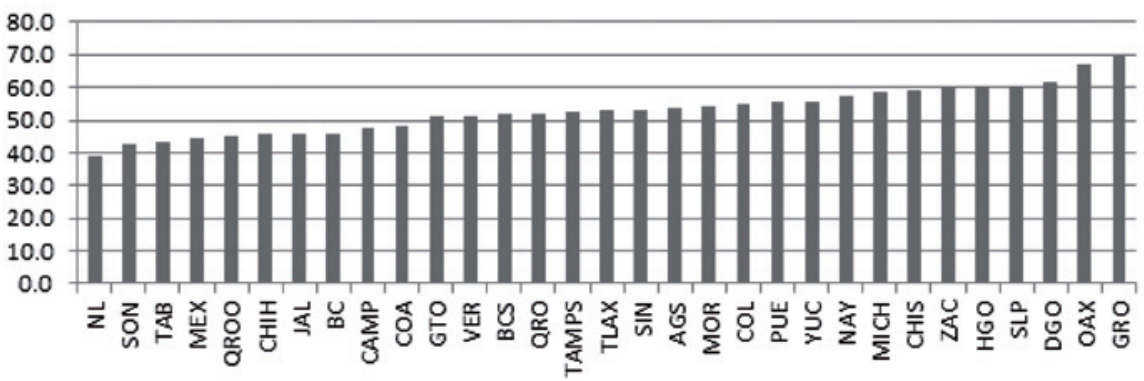

Fuente: Elaboración propia con base en datos del INEGI, Finanzas Públicas Estatales y Municipales

Lo que queremos destacar con lo anterior es que los Congresos locales, en comparación con la Cámara de Diputados, tienen un margen más reducido para realizar modificaciones al proyecto de presupuesto de egresos. Como se ha señalado ya, una parte de las transferencias son recursos etiquetados (aportaciones), es decir, no se pueden modificar. ${ }^{55}$ Entonces, para medir los cambios que el Congreso local haría al proyecto de presupuesto de egresos, hay que descontar las aportaciones. Los resultados que mostramos utilizan el indicador Función Presupuestaria Ejercida (FPE), que mide el porcentaje que representan los cambios respecto a los recursos potencialmente modificables del presupuesto de egresos. ${ }^{56}$

${ }^{55}$ Aunque se ha señalado que una parte de las aportaciones pueden ser determinadas por los actores locales. Véase Alejandra Ríos Cázares, "Instituciones políticas estatales y presupuesto. Las causas de las discrecionalidad”, op. cit., y Rogelio Hernández, El centro dividido, op. cit.

${ }^{56} \mathrm{La}$ fórmula es: $\mathrm{FPE}=\left(\frac{\mathrm{MF}}{\mathrm{MD}-\mathrm{A}}\right) * 100$, donde: $F P E$ es función presupuestaria ejercida; el numerador $M F$ representa el monto de recursos, que es modificado por la comisión dictaminadora; el denominador $M D$ es el presupuesto total dictaminado por la comisión; y $A$ representa las aportaciones recibidas por el estado. Más detalles metodológicos en Said Sánchez Martínez, "Gobiernos divididos y unificados: la función presupuestaria en los Congresos locales (2000-2012): Hidalgo, Guanajuato y San Luis Potosí”, Trabajo preparado para el Segundo Congreso Internacional de la Asociación Mexicana de Ciencia Política (AMECIP), septiembre de 2014, UAEM, Toluca, Estado de México. 


\section{El ejercicio de la función presupuestaria en los congresos locales: la etapa de aprobación}

Los siguientes datos muestran información para el período 2000-2012, en el cual existen 403 observaciones para los 31 estados. ${ }^{57}$ Por el momento, contamos con información para 336 observaciones, ${ }^{58}$ es decir, el $83.4 \%$. Sobre esta información preliminar realizaremos el análisis. Como hemos señalado, bajo gobiernos divididos se esperaría que el Congreso ejerciera de manera más activa su función presupuestaria que bajo gobiernos unificados. En términos operativos, realizará más modificaciones al proyecto de presupuesto de egresos del gobernador.

¿Qué nos indican los datos al respecto? Cuando consideramos los valores promedio de la FPE sin distinguir entre tipos de gobierno, se puede detectar un comportamiento oscilante. En la gráfica 2 podemos observar que, después de 3 años con un valor menor al $0.5 \%$, se presenta en el año fiscal 2003 un ascenso en el promedio de la FPE; a partir de entonces existen ascensos y descensos. El mayor valor promedio dentro del período de análisis corresponde al año fiscal 2005, con $2.35 \%$, seguido del año fiscal 2011 con un valor de $2.22 \%$ y del 2006 con $1.96 \%$. Al contextualizar estas cifras, en el caso de Presupuesto de Egresos de la Federación, la Cámara de Diputados ha hecho modificaciones que oscilan entre el $0.82 \%$ (año fiscal 2004) y el 7.76\% (año fiscal 2002)..$^{59}$ En el caso de los países de América Latina, la mayoría realiza cambios que representan porcentajes por debajo del $5 \% .{ }^{60}$

${ }^{57}$ Por cuestiones de disponibilidad de información, el período de análisis inicia en el año fiscal 2000 y termina en el año fiscal 2012. Los documentos (dictámenes) sobre los proyectos de presupuestos de egresos se obtuvieron mediante solicitudes de información a los Congresos locales.

${ }^{58}$ De las 336 observaciones, 146 (43.5\%) corresponden a los gobiernos divididos y 190 $(56.5 \%)$ a los gobiernos unificados.

${ }^{59}$ Laura Sour, op. cit. Otro estudio detectó oscilaciones entre el - $1 \%$ y el 6\%, véase Carpio, op. cit. Estas diferencias se derivan de la forma en que se operacionalizan las modificaciones.

${ }^{60} \mathrm{OCDE}$, "Prácticas presupuestarias en América Latina. Resultados de la encuesta de la OCDE 2006: Procedimientos y prácticas presupuestarias en los países de Latinoamérica”, 2007, Santiago de Chile, Seminario Regional de Política Fiscal. 
GRÁFICA 2

FPE promedio, 2000-2012

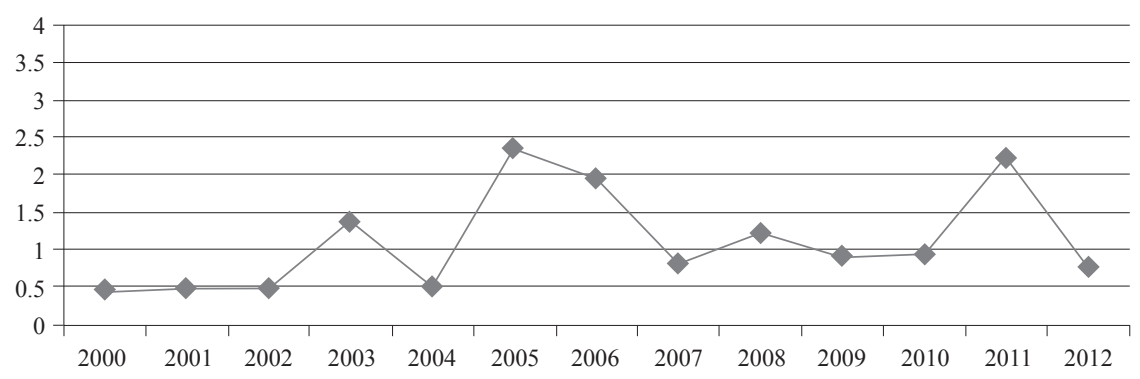

Fuente: elaboración propia con base en los dictámenes del proyecto de presupuesto de egresos de cada estado.

Al obtener el valor promedio de la FPE para cada categoría, observamos que los Congresos bajo gobiernos divididos realizaron más modificaciones que bajo los gobiernos unificados. En el primer caso, las modificaciones al proyecto de presupuesto del gobernador representaron el $2.11 \%$ de los recursos potencialmente modificables; en cambio, bajo gobiernos unificados el valor fue de $0.44 \%$.

GRÁFICA 3

Valores promedio de la FPE, 2000-2012

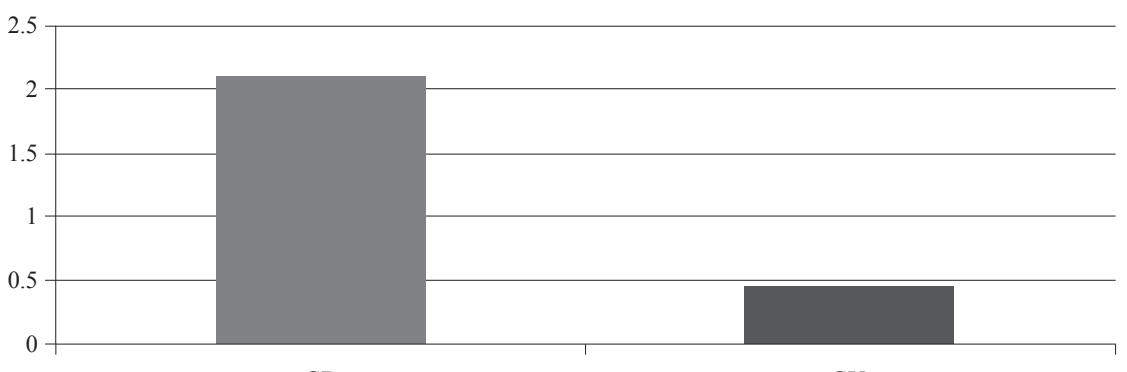

GD

GU

Fuente: Elaboración propia con base en los dictámenes del proyecto de presupuesto de egresos de cada estado.

Para ver lo que hay detrás de estos promedios, mencionamos algunos casos por año fiscal. Algunos de los Congresos bajo gobiernos divididos con mayores valores de la FPE son: San Luis Potosí, 40.7\% (año fiscal 2006); Jalisco, 26.9\% (año fiscal 2011); Guerrero, 25.3\% (año fiscal 
2011); Nayarit, 9\% (año fiscal 2003); y Querétaro, 8.3\% (año fiscal 2003). Como veremos más adelante, también hay años en los que no se realizaron modificaciones bajo gobiernos divididos. Por el lado de los Congresos bajo gobiernos unificados, también detectamos actividad: Hidalgo, 7.52\% (año fiscal 2005); Estado de México, 4.12\% (2012); Oaxaca, 5.93\% (2000), 0.14\% (2003), 0.03\% (2005), 0.54\% (2007); Puebla, 2.21\%(2011); Quintana Roo, 0.21\% (2002), 0.68\% (2004), 0.77\% (2005); y Veracruz, 0.16\% (2004), 1.14\% (2008), 3.17\% (2009), 0.11\% (2010) y 2.54\% (2012). Los Congresos de Coahuila y Tamaulipas no realizaron modificaciones en ninguno de los años del período de análisis.

Pero, ¿qué hay detrás de estas cifras? Para dar una respuesta, realizamos una breve comparación de tres casos considerados por otros estudios: Hidalgo, Guanajuato y San Luis Potosí ${ }^{61}$ En términos socioeconómicos, son estados similares; en términos políticos, en Guanajuato existe una fuerte oposición; Hidalgo es una estado relativamente no competitivo; y San Luis Potosí está en medio de ambos extremos. ${ }^{62}$

Antes de pasar a la cuestión de la función presupuestaria, es preciso destacar el contexto político de estos casos durante el período estudiado. Como podemos observar en el cuadro 1, en el estado de Hidalgo el partido del gobernador (PG) ha mantenido mayorías absolutas. En promedio, ha logrado el $63 \%$ de los escaños. El máximo porcentaje $(72 \%)$ se obtuvo en la LIX Legislatura; el menor (57\%) se logró recientemente, durante la LXI Legislatura. Aunque el número formal de partidos (NP) ha aumentado, no ha sido suficiente para afectar el predominio del PRI en el Congreso. Lo anterior se confirma en el número efectivo de partidos (NEP) ${ }^{63}$ el cual es el menor de los tres casos. En Guanajuato también identificamos la constancia de los gobiernos unificados, con excepción de la LVII Legislatura, la cual dictaminó el presupuesto para el año fiscal 2000 , dentro del período de estudio. El promedio de escaños del partido del gobernador es de 57.6\%. No obstante, tanto el NP como el NEP son

${ }^{61}$ Caroline Beer, op. cit.; Frederick Solt, op. cit.

${ }^{62}$ Criterios considerados por Caroline Beer, op. cit.

${ }^{63}$ Markku Laakso y Rein Taagepera, "Effective Number of Parties. AMeasure with Application to West Europe", en Comparative Political Studies, vol. 12, núm. 1, abril, 1979, pp. 3-27. 
mayores en comparación con el Congreso de Hidalgo. Por último, en San Luis Potosí encontramos un patrón de alternancia en el tipo de gobierno: de las 5 legislaturas, 3 han experimentado el gobierno divido. Lo anterior se refleja en el porcentaje promedio de escaños del partido del gobernador: $46 \%$. También observamos que este Congreso posee el mayor NEP promedio de los tres casos: 2.8 .

CUADRo 1

Características políticas de los Congresos locales

\begin{tabular}{|c|c|c|c|c|c|c|}
\hline Gobierno & Guanajuato & Legislatura & $N P$ & $N E P$ & $P G$ & Tipo de gobierno \\
\hline PAN & $1998-2000$ & LVII & 6 & 3.03 & 44 & GD \\
\hline \multirow[t]{2}{*}{ PAN } & $2001-2003$ & LVIII & 4 & 1.99 & 66 & GU \\
\hline & 2004-2006 & LIX & 5 & 2.77 & 53 & GU \\
\hline \multirow[t]{3}{*}{ PAN-PANAL } & $2007-2009$ & LX & 5 & 2.19 & 64 & GU \\
\hline & $2010-2012$ & LXI & 6 & 2.31 & 61 & GU \\
\hline & & PROMEDIO & 5.2 & 2.5 & 57.6 & \\
\hline Gobierno & Hidalgo & & $N P$ & $N E P$ & $P G$ & Tipo de gobierno \\
\hline \multirow[t]{2}{*}{ PRI } & $2000-2002$ & LVII & 4 & 2.20 & 62 & GU \\
\hline & $2003-2005$ & LVIII & 3 & 2.16 & 62 & GU \\
\hline \multirow[t]{2}{*}{ PRI-PVEM } & 2006-2008 & LIX & 5 & 1.83 & 72 & GU \\
\hline & 2009-2011 & LX & 5 & 2.27 & 63 & GU \\
\hline \multirow[t]{2}{*}{ PRI-PVEM-PANAL } & $2012-2014$ & LXI & 7 & 2.76 & 57 & GU \\
\hline & & PROMEDIO & 4.8 & 2.2 & 63 & \\
\hline Gobierno & San Luis Potosi & & $N P$ & $N E P$ & $P G$ & Tipo de gobierno \\
\hline \multirow[t]{2}{*}{ PRI } & $1998-2000$ & LV & 4 & 2.43 & 50 & GD \\
\hline & $2001-2003$ & LVI & 3 & 2.27 & 52 & GU \\
\hline \multirow[t]{2}{*}{ PAN } & $2004-2006$ & LVII & 5 & 2.69 & 41 & GD \\
\hline & 2007-2009 & LVIII & 6 & 2.71 & 56 & GU \\
\hline \multirow[t]{2}{*}{ PRI-PVEM-PASDC } & $2010-2012$ & LIX & 7 & 3.90 & 30 & GD \\
\hline & & PROMEDIO & 5 & 2.8 & 46 & \\
\hline
\end{tabular}

Fuente: Elaboración propia con base en solicitudes de información a los congresos locales. En el caso del partido en el gobierno, se usaron los datos electorales del CIDAC (www.cidac.org).

Dado que en Hidalgo y en Guanajuato han prevalecido los gobiernos unificados, se esperaría que el Congreso cuente con pocos incentivos para hacer modificaciones al proyecto de presupuesto de egresos. En contraparte, en San Luis Potosí, con cambios en el tipo de gobierno, se esperarían más modificaciones. 
En el estado de Hidalgo, el valor promedio del indicador es el más bajo de los tres casos: $0.66 \%$. De los trece años de estudio, sólo en dos se registraron modificaciones: $2001(0.9 \%)$ y 2005 (7.5\%). Estos porcentajes representaron reasignaciones por un monto de $340.4 \mathrm{mi}$ llones de pesos.

En el caso de Guanajuato se observa un porcentaje promedio mayor en comparación con Hidalgo: 1.3\%. De los trece años, en once se realizaron modificaciones al proyecto de presupuesto de egresos. El mayor porcentaje (4.1) corresponde al año fiscal de 2001, en tanto que los años fiscales sin modificaciones fueron 2011 y 2012. Las cambios realizados durante el período representaron un monto de 1,937.3 millones de pesos.

En concordancia con los supuestos, San Luis Potosí acumula el mayor valor promedio: 4.4\%. El año fiscal con el mayor porcentaje es 2006: 40.7\%. En contraparte, los años sin modificaciones fueron 2001, 2002, 2003, 2011 y 2012. Los cambios realizados representaron un monto de 2,847.6 millones de pesos. Cuando analizamos en detalle los tipos de gobierno, vemos que en la LVI (2001-2003) Legislatura (gobierno unificado) no se realizaron modificaciones en los montos; sin embargo, en la LVIII (2007-2009) Legislatura (gobierno unificado) encontramos una FPE promedio de $1.7 \%$, registrándose el mayor valor (4.8\%) en el año fiscal 2007. En contextos de gobierno dividido, en la LVII (2004-2006) Legislatura se registró el mayor valor de la FPE: 17.3\%; en contraparte, en la LIX (2010-2012) Legislatura sólo en un año se hicieron modificaciones (2010).

De manera general, los datos anteriores muestran que bajo gobiernos divididos el Congreso realiza más modificaciones, en promedio, que bajo gobiernos unificados. No obstante, como hemos visto, bajo gobiernos unificados también hay actividad; por otro lado, cuando hay cambios entre tipos de gobierno, como en San Luis Potosí, observamos que el gobierno dividido no garantiza modificaciones al presupuesto ni el unificado implica una aprobación automática.

¿Qué representan estos datos en términos más concretos? Al hacer un análisis más detallado es posible identificar los temas que son modificados por la comisión dictaminadora. En otras palabras, se observan los temas en los que el Congreso no estuvo de acuerdo con el diseño de gasto del gobernador y aquellas áreas que fueron consideradas priori- 
tarias por este órgano. Para mostrar lo anterior, nos centraremos en el caso de Guanajuato, mencionando un par de años. ${ }^{64}$

En el presupuesto del año fiscal 2000, bajo gobierno dividido, se reasignaron alrededor de 82.7 millones de pesos. Las reducciones realizadas se justificaron bajo los "principios de racionalidad y austeridad que deben regir en el ejercicio del gasto público". ${ }^{65}$ En el cuadro 2 observamos la distribución de los cambios. La reducciones tocan varios ámbitos y a los tres poderes; los temas que fueron beneficiados por los aumentos son principalmente de orden social y de inversión física. Incluso se detalla el destino de los aumentos en cada tema; por ejemplo, se estableció que del aumento para la Secretaría de Desarro1lo Urbano y Obras Públicas, 10 millones de pesos fueran para la construcción de carreteras, 5 millones para caminos rurales y 5 millones para el manteniendo de la red de carreteras. En el caso de los aumentos para la Salud, su destino se enfocó en la capacitación del personal y en el equipamiento de determinados hospitales. Como se aprecia, existe un déficit de alrededor de 8.2 millones de pesos, monto del que no se señalan más detalles en el dictamen.

Por último, mencionamos el año fiscal 2001. A pesar de existir gobierno unificado, se registró un cambio mayor al proyecto de presupuesto de egresos del gobernador. Los recursos modificados representaron alrededor de 327 millones de pesos; los temas que experimentaron recortes fueron muy variados. En cambio, del lado de los aumentos, los favorecidos tuvieron que ver en mayor medida con la seguridad pública, la impartición de justicia y el desarrollo agropecuario. También se pueden encontrar especificaciones en los aumentos; por ejemplo, del aumento de 9.1 millones para la Procuraduría General de Justicia, se estableció que 3 millones fueran destinados a programas de prevención del delito y atención a víctimas; 2 millones para el fortalecimiento de los servicios periciales; un millón para rediseñar el procedimiento operativo del servicio pericial; y 3.1 millones para el programa de combate a los delitos del campo. Del aumento de 23.8 millones de pesos para la

${ }^{64}$ Mostramos este caso debido a la disponibilidad de información.

${ }^{65}$ Dictamen de las Comisiones Unidas de Gobernación y Puntos Constitucionales, de Justicia y de Hacienda y Revisora de la Contaduría Mayor de Hacienda, relativo a la Ley del Presupuesto General de Egresos, año fiscal 2000, Congreso del Estado de Guanajuato, 22 de diciembre, 1999, p. VI. 
Secretaría de Desarrollo Agropecuario, se especificó que: 500 mil fueran destinados al Programa de Apoyo a la Micro Empresa Rural; 1.1 millones al Programa de Apoyo a Productores de Bajos Recursos; 500 mil al Programa de Fomento Lechero; 1.5 millones al Programa de Mejoramiento Genético; 500 mil al Programa de Ganado Mejor; un millón al Programa de Establecimiento de Praderas y Agostaderos; 2 millones al Programa de Ovino-Caprino; 3 millones al Programa de Modernización de Mercados Agropecuarios, etcétera. Nuevamente, también se detecta un déficit derivado de los cambios, aunque no se especifican detalles. ${ }^{66}$

CUADRO 2

Reasignaciones al proyecto de presupuesto de egresos, año fiscal 2000

\begin{tabular}{|c|c|c|c|}
\hline Tema & Reducción & Tema & Aumento \\
\hline Ramo 23 & $1,000,000.00$ & Coordinadora de Turismo & $500,000.00$ \\
\hline $\begin{array}{l}\text { Radio y Televisión de } \\
\text { Guanajuato }\end{array}$ & $2,000,000.00$ & $\begin{array}{l}\text { Instituto de la Mujer } \\
\text { Guanajuatense }\end{array}$ & $2,500,000.00$ \\
\hline $\begin{array}{l}\text { Comunicación Social del } \\
\text { Poder Ejecutivo }\end{array}$ & $2,000,000.00$ & Instituto de Ecología & $2,500,000.00$ \\
\hline Ramo 24 & $2,650,000.00$ & Instituciones Educativas & $3,000,000.00$ \\
\hline $\begin{array}{l}\text { Centro de Información } \\
\text { Guanajuato }\end{array}$ & $3,000,000.00$ & $\begin{array}{l}\text { Comisión Estatal del } \\
\text { Deporte }\end{array}$ & $3,800,000.00$ \\
\hline $\begin{array}{l}\text { Sistema Estatal de } \\
\text { Financiamiento al } \\
\text { Desarrollo del Estado }\end{array}$ & $3,000,000.00$ & ONG's & $4,000,000.00$ \\
\hline Poder Legislativo & $3,500,000.00$ & $\begin{array}{l}\text { Instituto de Seguridad y } \\
\text { Servicios Sociales }\end{array}$ & $7,000,000.00$ \\
\hline $\begin{array}{l}\text { Coordinadora de Fomento } \\
\text { al Comercio exterior }\end{array}$ & $4,000,000.00$ & Salud & $7,050,000.00$ \\
\hline $\begin{array}{l}\text { Coordinación General } \\
\text { para el Desarrollo } \\
\text { Regional }\end{array}$ & $5,500,000.00$ & Universidad de Guanajuato & $8,000,000.00$ \\
\hline $\begin{array}{l}\text { Instituto Electoral del } \\
\text { Estado de Guanajuato }\end{array}$ & $8,468,000.00$ & $\begin{array}{l}\text { Creación de los Centros } \\
\text { de Atención a la Violencia } \\
\text { Intrafamiliar }\end{array}$ & $10,000,000.00$ \\
\hline Poder Judicial & $25,000,000.00$ & $\begin{array}{l}\text { Secretaría de Desarrollo } \\
\text { Urbano y Obras Públicas }\end{array}$ & $20,000,000.00$ \\
\hline Total & $60,118,000.00$ & & $68,350,000.00$ \\
\hline
\end{tabular}

Fuente: elaboración propia con base en el dictamen del presupuesto de egresos, año fiscal 2001.

${ }^{66}$ Dictamen de las Comisiones Unidas de Gobernación y Puntos Constitucionales, de Justicia y de Hacienda y Revisora de la Contaduría Mayor de Hacienda, relativo a la Ley del Presupuesto General de Egresos, año fiscal 2001, Congreso del Estado de Guanajuato, 21 de diciembre, 2000. 


\section{CUADRO 3}

\section{Reasignaciones al proyecto de presupuesto de egresos, año fiscal 2001}

\begin{tabular}{l} 
Tema \\
\hline Gubernatura \\
Promotora de Infraestructura \\
Educativa \\
Instituto de Educación \\
Permanente \\
Varios programas de la Secretaría \\
de Desarrollo Social y Humano
\end{tabular}

Secretaría de Desarrollo

Económico

Varios proyectos de la

Secretaría de Obra Pública

Varios ramos generales

Secretaría de Gobierno

\section{,}

\begin{tabular}{|c|c|c|}
\hline $12,413,650.00$ & $\begin{array}{l}\text { Administración Pública } \\
\text { Paraestatal }\end{array}$ & $1,000,000.00$ \\
\hline $27,486,930.00$ & Instituto de Salud Pública & $1,000,000.00$ \\
\hline $46,368,800.00$ & $\begin{array}{l}\text { Reasignación Administración } \\
\text { Pública Paraestatal }\end{array}$ & $1,000,000.00$ \\
\hline \multirow[t]{15}{*}{$225,000,000.00$} & Tribunal Contencioso & $2,000,000.00$ \\
\hline & Administrativo & \\
\hline & $\begin{array}{l}\text { Instituto de Financiamiento } \\
\text { e Información para }\end{array}$ & $2,500,000.00$ \\
\hline & la Educación & \\
\hline & $\begin{array}{l}\text { Sistema Estatal para el } \\
\text { Desarrollo Integral }\end{array}$ & $3,000,000.00$ \\
\hline & Instituto de Ecología & $3,500,000.00$ \\
\hline & $\begin{array}{l}\text { Procuraduría General } \\
\text { de Justicia }\end{array}$ & $9,100,000.00$ \\
\hline & $\begin{array}{l}\text { Reasignación Secretaría } \\
\text { de Obra Pública }\end{array}$ & $11,836,350.00$ \\
\hline & Universidad de Guanajuato & $12,000,000.00$ \\
\hline & Poder Judicial & $13,200,000.00$ \\
\hline & Artículo transitorio para & $20,000,000.00$ \\
\hline & Instituto Electoral & \\
\hline & Secretaría de Desarrollo & $23,854,500.00$ \\
\hline & Agropecuario & \\
\hline & Secretaría de Seguridad Pública & $225,000,000.00$ \\
\hline $327,069,380.00$ & Total & $331,700,580.00$ \\
\hline
\end{tabular}

$1,000,000.00$ Ramos generales

2,000,000.00 Instituto Estatal de la Cultura

5,000,000.00 Procuraduría de los Derechos Humanos

7,800,000.00 Reasignación Secretaría

de Desarrollo Económico

Temamento

$$
\begin{array}{r}
49,730.00 \\
660,000.00
\end{array}
$$

$1,000,000.00$

$1,000,000.00$

Administración Públic 
nes para el año fiscal 2001 representaron el $4.11 \%$ de los recursos potencialmente modificables. Pero cuando comparamos el monto modificado con el presupuesto de ciertas áreas, obtenemos diversas perspectivas. Por ejemplo, los cambios representaron los siguientes porcentajes: el 27\% del presupuesto del Instituto de Salud Pública; el 68\% del presupuesto de la Secretaría de Gobierno; el 145\% de la Secretaría de Seguridad Pública; y el 486\% del Instituto de Vivienda del Estado. ${ }^{67}$

En síntesis, la evidencia hasta aquí mostrada matiza la fortaleza institucional de los gobernadores en la política presupuestal, específicamente en la fase de la aprobación (control ex ante). Su supremacía formal no es suficiente para someter de manera absoluta al Congreso: ni en una política tan importante como lo es la presupuestal ni cuando poseen mayorías legislativas. De los tres casos, el Congreso de San Luis Potosí, con más tiempo bajo gobiernos divididos, ha realizado las mayores modificaciones al proyecto de presupuesto de egresos del gobernador; no obstante, también se registra actividad en los Congresos bajo gobiernos unificados. Destaca el caso de Guanajuato, en el que no sólo hay modificaciones, sino que también se detalla la forma en que serán reasignados los montos.

\section{Reflexiones finales}

El objetivo de este ensayo fue mostrar que los Congresos locales no son actores marginales en la política presupuestaria, específicamente en la fase de la aprobación. Nos enfocamos en la función presupuestaria porque es una de las más importantes que realiza el poder Legislativo: es el poder del tesoro que restringe la acción del Ejecutivo en términos de gasto. Además, es la política matriz para todas las demás políticas públicas, ya que en el presupuesto de egresos se determinan los recursos para su operación.

Si bien es cierto que los gobernadores son formalmente más fuertes que los Congresos, una parte de esta fortaleza se deriva de las caracte-

${ }^{67}$ Cálculos propios obtenidos del análisis del Dictamen de las Comisiones, op. cit. 
rísticas del juego bilateral de veto en el que participan. En éste, el Ejecutivo es quien mueve la primera pieza; sin embargo, el carácter reactivo de la Legislatura no la hace un actor sin importancia en esta relación, ya que se involucra en la fase de aprobación, especialmente al modificar el proyecto de gasto. Las características de su reacción dependen tanto de aspectos formales como de aspectos partidistas.

Para ambos tipos de gobierno (unificado y dividido), la evidencia contrasta con la idea de los gobernadores como virreyes sin contrapesos. Incluso contar con mayorías absolutas no les garantiza un respaldo mecánico por parte del Congreso, como se esperaría. Los Congresos locales no son pasivos en la política de gasto. Los resultados preliminares muestran que, en efecto, bajo gobiernos divididos el Congreso realiza más modificaciones en promedio que bajo gobiernos unificados. Esto no debe pasar por alto las modificaciones que también se realizan bajo gobiernos unificados. Al exponer el caso de Guanajuato, hemos visto que no sólo hay actividad en términos de reasignaciones en el proyecto de presupuesto de egresos, sino que también se detallan las áreas que recibirán los recursos, lo que muestra las preferencias de los legisladores.

Aunque el indicador FPE no capta las negociaciones previas al dictamen del proyecto ni otras prácticas informales, el hecho de que se detecten modificaciones muestra la pluralidad de preferencias en torno al gasto que existe entre el Congreso y el Ejecutivo. Insistimos, no buscamos minimizar las deficiencias democráticas que existen a nivel subnacional. Tampoco queremos sobrevalorar la capacidad del Congreso para incidir en la política pública al realizar cambios al presupuesto. Buscamos resaltar que, a pesar de las mayores facultades formales que poseen los gobernadores, los Congresos locales están mostrando actividad en el ejercicio del poder del tesoro, específicamente en la fase de aprobación. Esta actividad nos invita no sólo a repensar su relación frente a los gobernadores, sino también a analizarla con mayor detalle y a tomar cierta distancia de los lugares comunes. 